TAO, Vol. 15, No. 3, I-IV, September 2004

\title{
Preface to the Special Issue on Earthquake Precursors
}

\author{
Yi-Ben Tsai and Jann-Yenq Liu
}

\section{PREFACE}

"Are earthquakes predictable?" is a question hotly debated in the earthquake science community for some time. Its answer begs for another question "Are there credible earthquake precursors?" After the disastrous 1995 Kobe earthquake, the former Science and Technology Agency (STA) of Japanese Government sponsored, in response to these questions, an Integrated Earthquake Frontier Project. Two institutions, the Institute of Physical and Chemical Research (RIKEN) and the National Space Development Agency of Japan (NASDA) were asked to carry out for five years feasibility studies on possible use of electromagnetic phenomena for short-term earthquake prediction. Since 1996 the RIKEN group formed the International Frontier Research Project on Earthquakes (IFREQ) to study earth electric current and related phenomena. Meanwhile, the NASDA team formed the Earthquake Remote Sensing Frontier Project to perform an overall study of seismo-electromagnetic phenomena occurring in the lithosphere, atmosphere and ionosphere.

Similarly, after the disastrous 1999 Chi-Chi earthquake, scientists in Taiwan were intrigued by instrumental observations of conspicuous ionospheric and geomagnetic disturbances before the earthquake. They decided to undertake in 2002 a four-year multidisciplinary program to pursue integrated Search for Taiwan Earthquake Precursors (iSTEP), under the support of the Ministry of Education. Since these organized programs in Japan and Taiwan are unique in the atmosphere of ambivalence toward earthquake prediction research, we decide to publish this special issue to highlight some significant results obtained in recent years by scientists both inside and outside these programs.

We have included fourteen papers in this special issue. Broadly speaking, the first group of three papers reviews major results of the post-Kobe Integrated Earthquake Frontier Project. The second group of four papers presents post-Chi-Chi studies of electromagnetic precursors of earthquakes in Taiwan. The third group of two papers presents models on possible physical mechanisms for electromagnetic variations associated with large earthquakes. The fourth group of three papers devolves on seismicity patterns before and after large earthquakes. One paper explores potential applications of the novel space-borne radar interferometry for detecting regional crustal deformation before major earthquakes. The final paper presents preliminary results of the post-Chi-Chi iSTEP program.

In the first group of papers, Uyeda et al. report main results from the IFREQ. They review state of the art of short-term earthquake prediction, in particular recent progress in using seismoelectromagnetic phenomena. The IFREQ demonstrated the existence of pre-seismic ultra-low frequency (ULF) geoelectric potential changes and verified the precursory signatures of ULF geomagnetic variations before some earthquakes. Hayakawa and his NASDA team report the current achievements of NASDA's Earthquake Remote Sensing Frontier Project in last five years. They performed an overall study of seismo-electromagnetic phenomena occurring not only in the lithosphere, but also in the atmosphere and ionosphere. They further proposed a 
lithosphere-atmosphere-ionosphere coupling system for short-term earthquake prediction. Most importantly, they found convincing evidence for seismo-ionospheric perturbations in the subionospheric VLF/LF signals. Finally, Hattori reviews the ULF geomagnetic changes associated with large earthquakes. He first summarizes earlier observational results and then presents the latest results, especially on the existence of ULF emissions using sophisticated signal processing techniques.

In the second group of four papers, Chen et al. examine variations of geomagnetic total field recorded by eight magnetometer stations and correlate them with occurrences of $M \geq 6.0$ earthquakes in Taiwan from 1989 to 2001. They found that the annual change rates of the field strength reduce down to a zero isoporic value of $\pm 5 \mathrm{nT} / \mathrm{yr}$ after 1997. The following $\mathrm{M} \geq 6.0$ earthquakes often occur within the zero isoporic zone (ZIZ). Liu et al. report ionospheric foF2 and TEC anomalies during $M \geq 5$.0 Taiwan earthquakes. They have correlated the variations in the critical frequency foF 2 recorded by an ionosonde, and in the total electron content (TEC) observed by a network of five ground-based global positioning system (GPS) receivers, with the occurrences of $144 \mathrm{M} \geq 5.0$ earthquakes in Taiwan during 1997-1999. The results show that the foF2 and TEC yield similar trends, and often concurrently register anomalous depressions about four days before the earthquakes. Chen et al. present results of statistical tests on the pre-earthquake ionospheric anomalies. They show that the anomalous depressions of foF2 are significant within 1-5 days before $\mathrm{M} \geq 5.0$ earthquakes in Taiwan area during 1994-1999 (Liu et al. 2003). They propose two statistical tests on the foF2 anomaly as precursor of earthquakes based on criteria including the successful rate, alarm rate, probability gain, and $\mathrm{R}$ score. In the last paper of this group, Kamogawa et al. make observations of atmospheric electric field (AEF) in Taiwan during last three years. They identify clear variations in atmospheric electric field before the earthquake of 31 March, 2002.

In the third group of papers, Pulinets summarizes recent advances in the understanding of seismo-ionospheric coupling which are covered in three parts: physical mechanism, main phenomenological features of ionospheric variations associated with earthquakes, and their statistical properties useful for practical applications. Freund et al. demonstrate the stress-induced changes in electrical conductivity of igneous rocks and the generation of ground currents. They have studied how the electrical conductivity of igneous rocks changes under stress and what charge carriers are involved. They show that quartz-rich granite and quartz-free anorthosite can generate electronic charge carriers ( $\mathrm{p}$-holes) when subjected to stress. They propose such stress-induced generation of charge carriers and their outflow as the basic process by which ground currents in the Earth's crust can be generated. The arrival of p-holes at the Earth's surface leads to changes in the ground potential, which then induce ionospheric perturbations. They further propose that the build-up of high electric fields at the ground surface can cause ionization of the air, hence the ion emission and corona discharges.

The fourth group of papers are concerned with seismicity pattern changes before and after large earthquakes. Huang applied a statistical method, the Region-Time-Length (RTL) algorithm, which takes into account the magnitude, occurrence time and place of earthquakes, to investigate seismicity pattern changes before large earthquakes. Based on the RTL algorithm, he quantified both the temporal and spatial characteristics of seismicity pattern changes in various tectonic regions. He pointed out that the duration of the subsequent stage of seismic 
activation lasted from several months to 1.5 years and the linear dimension of the quiescence zone could reach a few hundred kilometers (several time of the rupture dimension of the mainshock). Chen et al. makes a statistical assessment of Chi-Chi aftershock hazards. They aim to develop a real-time hazard assessment of large aftershocks following a major earthquake for disaster relief and rescue efforts. They used the Reasenberg-Jones (RJ) model to assess the aftershock hazards from the 1999 Chi-Chi earthquake. A probabilistic aftershock hazard map (PAHM) is also constructed for likely spatial distribution of $\mathrm{M} \geq 5.0$ aftershocks over the study region. Chan and Ma use the spatially heterogeneous slip dislocation models from seismic waveform inversion of several moderate to large earthquakes in the past decade in Taiwan area, to calculate the conditions of stress transfer associated with the aftershock distributions. The stress change calculation for optimum orientation planes after the mainshock shows great consistency of the positive stress change to the aftershock distribution.

Chang et al. explore potential applications of space-borne radar interferometry for detecting precursory crustal deformation before large earthquakes in Taiwan. This paper reports recent results from applying the novel radar interferometry technique to detect crustal deformation in Taiwan. They classified the crustal deformation events into intense and gentle events. Results from five deformation events show that the radar interferometry is a high resolution tool useful for monitoring crustal deformation of different nature.

The last paper by Tsai et al. presents an overview of preliminary results of the iSTEP program on integrated search for Taiwan earthquake precursors. The multidisciplinary program includes five major components aiming at identifying potential seismological, geomagnetic, geodetic and ionospheric precursors, respectively and to perform statistical tests on any identified precursors. Since the program's inception in April 2002, some encouraging preliminary results are obtained. These includes precursory $\mathrm{P}$ wave travel-time changes occurring about six years before the Chi-Chi earthquake, identifiable geomagnetic changes two years before $M \geq 6$ earthquakes, consistent ionospheric anomalous depressions about three to five days before $M \geq 5$ earthquakes. They have also developed a high-resolution Radar interferometric method for monitoring crustal deformation. A method for forecasting aftershock distribution on the basis of stress transfer is also successfully tested on the Chi-Chi earthquake sequence. Aided by the newly installed geomagnetic and ionospheric networks they are hopeful about identifying more earthquake precursors and developing relevant physical mechanisms in the remaining two years of the iSTEP program.

In summary, the papers in this issue, even though still preliminary, apparently have identified several promising precursory changes, ranging from a few years to a few days prior to large earthquakes. Most authors are hopeful that they can identify more earthquake precursors from observations of new instrumental networks in the near future. In the meantime, some of them continue to pursue relevant physical mechanisms. Since researchers working in similar fields are spread in different countries, such as Taiwan, Japan, China, and the U.S. international exchange and cooperation with each other are particularly important. Through integrated search for the earthquake precursors and positive establishment of related physical mechanisms, we hope to lay foundation for eventual earthquake prediction. We can not give up hope for earthquake prediction even the road may be long and hard, simply because the societal stakes are too high in earthquake countries. 
Guest Editors:

Jann-Yenq Liu

Institute of Space Science, National Central University, Chung-Li, Taiwan

E-mail: jyliu@jupiter.ss.ncu.edu.tw

Yi-Ben Tsai

Institute of Geophysics, Chung-Li, Taiwan

E-mail: ybtsai@geps.gep.ncu.edu.tw 